\title{
A Reference Calorimeter for Laser Energy Measurements*
}

\author{
E. D. West, ${ }^{* *}$ W. E. Case, ${ }^{* *}$ A. L. Rasmussen, ${ }^{* *}$ and L. B. Schmidt**
}

Institute for Basic Standards, National Bureau of Standards, Boulder, Colorado 80302

(September 3, 1971)

\begin{abstract}
Principles and detailed procedures are described for measuring laser energy and power in terms of electrical energy based on voltage, resistance, and frequency standards. The construction of a small isoperibol calorimeter used for the measurements is described. The calorimeter will accommodate 0.01 to $20 \mathrm{~J}$ and $4 \times 10^{-5}$ to $1 \mathrm{~W}$ cw and is limited to a maximum pulse intensity of $0.1 \mathrm{~J} / \mathrm{cm}^{2}$. The standard deviation of comparison measurements using two calorimeters and a beam splitter is 0.08 percent when the smaller energy input is not less than $0.3 \mathrm{~J}$. The estimated limits of systematic error for one calorimeter are \pm 1.0 percent of the laser energy measured by the calorimeter.
\end{abstract}

Key words: Calorimetry; laser; laser calorimetry; laser energy measurement; laser power measurement.

\section{Introduction}

Calibration of a device for laser power and energy measurements is just the process by which the output of the device is translated into watts or joules based on standards maintained by the National Bureau of Standards. Evidently, methods of measurement which can compare laser outputs directly to the basic electrical standards offer considerable advantages by reducing the number of steps in the calibration process and the associated propagation of errors. These more direct methods of measurements have been the object of a program conducted at NBS $[1,2,3,4] .{ }^{1}$ The main thrust of the work has been to apply calorimetric methods to the measurement of laser power and energy, and, through the use of beam splitters, to provide monitored beams of known energy for calibrating other measuring devices. More recently the work has been directed toward calorimeters which are simple enough for non-experts to operate, rugged enough to ship between laboratories, but accurate enough to provide a means of referring laser power and energy to the NBS electrical standards - a reference calorimeter. In this paper we describe principles and procedures for referring laser power and energy to electrical standards by means of a group of calorimeters, which we designate the C-series*, meeting most of the above requirements. It is contemplated that calorimeters of this type will be the basis of a service to calibrating laboratories to check on their overall accuracy and precision, much in the manner of the present system used with the standards of mass $[5,6]$.

\footnotetext{
* Contribution of the National Bureau of Standards. Not subject to copyright.

** Quantum Electronics Division, National Bureau of Standards, Boulder, Colo. 80302

${ }^{1}$ Figures in brackets indicate the literature references at the end of this paper.

*There have been three designs thus far, designated C1, C2, and C3. The first was a prototype. The principal difference between C2 and C3 is that C3 has an incomplete outer shield and a conical mirror so that the calorimeter can accommodate laser beams of larger diameter.
}

Besides reducing the number of steps in the calibration process, calorimetry has other advantages. Calorimetry can be used to measure the energy in a pulse or, by the use of a suitable timer, a wide range of average power levels. A calorimeter properly designed and operated can make a valid comparison of energies independent of the time required to put the energy into the calorimeter. It is valid to compare energy in a pulse to cw energy put in over a 5 minute period, for example, or to compare a laser pulse to an electrical input of 10 to $300 \mathrm{sec}$ duration. To conform with calorimetric tradition, we should probably restrict the term calorimeter to devices which can perform such time-dependent energy comparisons and relegate other so-called calorimeters to the category of thermal detectors. It is a technique that has been in use for about 100 years, so that a great deal of information on the design and operation of calorimeters is available $[7,8,9,10]$. Calorimeters can be shaped to approximate a total absorber, to reduce the dependence of the calibration on the wavelength of the laser radiation. They can be constructed so that the calibration factor does not depend on where the laser beam strikes the calorimeter. This geometric variation of the calibration factor is rather common-not only in thermal devices, such as bolometers, thermopiles, and conduction calorimeters, but also in devices which respond to light quanta, such as photoelectric tubes. A calorimeter will retain its calibration factor for a very long time, unless it is damaged by a gross error, such as exceeding the maximum ratings for power density or energy. Using a beam-splitter, intercomparison and calibration of energy measuring calorimeters can be made independent of the laser stability, either in cw power or the energy of single or multiple pulses.

The requirements for laser power and energy measurements are extremely varied-wavelengths from $0.4 \mu \mathrm{m}$ to $30 \mu \mathrm{m}$, continuous power levels from $10^{-6}$ to $10^{3} \mathrm{~W}$, pulse powers in gigawatts, and 
energies from $10^{-3}$ to $10^{3} \mathrm{~J}$ in single or repetitive pulses. Calorimeters are probably adaptable to a greater range of these requirements than other methods of laser power and energy measurements, but they too must be designed for a restricted portion of the problems. The practical operating parameters for the C-series calorimeters are summarized in table 1 .

Although this paper describes a particular calorimeter in detail, its main purpose is to describe apparatus design and experimental techniques which any laboratory can follow to refer laser power and energy measurements to electrical standards.

\section{The Theory of the Measurement}

The C-series calorimeters, in common with the liquid cell calorimeter [3], are of the isoperibol type; that is they operate in an environment in which the temperature does not change with time. The theory for this type of calorimeter has been worked out in detail from its basis in the First Law of Thermodynamics and a generalized boundary-value heat flow problem describing isoperibol calorimeters [11]. The application of the theory to data reduction for temperatures taken at equal time intervals has been worked out for both a least squares computer program and a manual approximation [12]. The theory is summarized in the equation

$$
W=E\left[T_{F}-T_{I}+\epsilon \int_{t_{l}}^{t_{F}}\left(T-T_{\infty}\right) d t\right]
$$

where $W$ is the thermodynamic work done on the calorimeter, either by a laser beam or by a calibrating electrical current; $E$ is the energy equivalent determined with a known work quantity; $T$ is the temperature (or a quantity linearly related to the temperature [12]); $\epsilon$ is the cooling constant described below; and $t$ is time. The work quantity $W$ is the actual work done by the laser beam; an additional measurement is required to determine any power or energy in the beam which is scattered back out of the calorimeter. The

TABLE 1. Operating parameters of C3 calorimeters

\begin{tabular}{|c|c|}
\hline 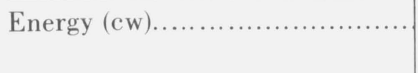 & $\begin{array}{l}0.03 \text { to } 3 \mathrm{~J}(0.01 \text { to } 20 \mathrm{~J} \text { slightly } \\
\text { less accurate })\end{array}$ \\
\hline Wavelength (with BK7 window)... & 0.4 to $2 \mu \mathrm{m}$ \\
\hline Aperture $(\max$ beam size $) . \ldots \ldots \ldots$ & $2 \mathrm{~cm}$ \\
\hline $\begin{array}{l}\text { Cooling constant (reciprocal time } \\
\text { constant) }\end{array}$ & $0.003 \mathrm{~s}^{-1}$ \\
\hline Power range $(\mathrm{cw}) \ldots \ldots \ldots \ldots$ & $4 \times 10^{-5}$ to $2 \mathrm{~W}$ \\
\hline $\begin{array}{l}\text { Max pulse intensity (pulse } \\
\quad<10^{-3} \mathrm{~s} \text { ) }\end{array}$ & $0.1 \mathrm{~J} / \mathrm{cm}^{2}$ \\
\hline $\begin{array}{l}\text { Precision (standard deviation of } \\
\text { an electrical calibration) }\end{array}$ & 0.2 percent \\
\hline Systematic error..... & less than 1.0 percent \\
\hline
\end{tabular}

quantity in brackets is traditionally called the corrected temperature rise $\Delta T_{c}$ and is determined from observations of the temperature over the time of the experiment. The quantity $E\left(T_{F}-T_{I}\right)$ is very nearly the change in the internal energy of the calorimeter and the product $E \epsilon$ times the integral is very nearly the heat exchanged between the calorimeter and the surroundings. The subscripts $I$ and $F$ refer to the initial and final rating periods, as outlined below. The initial and final temperatures must be observed during rating periods as defined by equation (2). The convergence temperature $T_{\infty}$ is the temperature observed an infinite time, in a practical sense, after a disturbance of the calorimeter.

The other equation essential to the measurement $[11,12,13]$, is the following:

$$
\frac{d T}{d t}=-\epsilon\left(T-T_{\infty}\right)
$$

When the temperature obeys this equation the calorimeter is said to be in a rating period. The measurement requires that rating periods both precede and follow the work input to the calorimeter. Two equationsone for each rating period-can be solved for the convergence temperature $T_{\infty}$ and the cooling constant $\epsilon$. Obviously $T_{\infty}$ is the temperature when its rate of change $d T / d t$ is zero. It is shown [11] that $\epsilon$ is the smallest eigenvalue of the heat flow problem; it can be varied by changing the thermal contact between the calorimeter and its constant-temperature environment, by removing air in the space around the calorimeter, for example.

It is important to note that equations (1) and (2) take into account the fact that the calorimeter has an opening for the laser beam and therefore must be somewhat influenced by objects in the room. Only the variation during an experiment of the radiation from the room will have an effect and this variation will appear in the random error of the calibration factor. For measurements at low light levels it may be preferable to keep objects in the room, such as supervisors, in fixed positions.

The use of equation (1) with actual data requires averaging techniques to achieve the highest accuracy and precision $[12,13]$. We reduce data for the Cseries and other isoperibol calorimeters by a simple least squares computer program [12]. Alternatively it may be accomplished manually. A simple manual method sacrifices very little precision or accuracy [12].

The least squares program in effect fits the integral of equation (2) to the data in the initial and final rating periods to obtain the best values for $T_{F}, T_{I}, \epsilon$, and $T_{\infty}$ in equation (1). The integration is carried out by the trapezoidal rule.

Equation (1) contains all of the quantities used in the computation of the energy equivalent $E$ and is therefore the starting point for the analysis of errors in electrical calibration experiments.

Calorimetry compares laser energy actually absorbed to electrical energy. The energy in the beam as 
it strikes the calorimeter is greater than the energy absorbed because of the losses in the window on the calorimeter and the small reflectance of the calorimeter proper. If $W_{l}$ is the incident laser energy and $\tau_{w}$ is the transmission of the window, then $\tau_{w} W_{l}$ is the energy incident on the calorimeter proper, which absorbs a fraction $\alpha$, so that the work quantity $W$ in equation (1) is just $\alpha \tau_{w} W_{l}$. As we measure it, the quantity $\alpha$ also allows for a possible small heat exchange term. When the laser beam strikes the absorbing surface, that surface will be heated above the corresponding temperature in the electrical experiment. There will be a corresponding increase in the heat loss by thermal radiation over that accounted for by isoperibol theory. We call this the excess thermal radiation and treat it along with the absorption. Writing $\Delta T_{c}$ for the corrected temperature rise, we obtain the laser energy incident on the calorimeter window in terms of observable quantities

$$
W_{l}=\frac{E \Delta T_{c}}{\tau_{w} \alpha} .
$$

The determination of the energy in the laser beam depends on the determination of the four quantities on the right of equation (3). This paper will be concerned mainly with these quantities. Equation (3) contains all the quantities required to compute the energy from a laser beam and is therefore the starting point for the analysis of errors in laser experiments.

Systematic errors may also be due to inadequacies of the theory of the measurement. Problems related to the adequacy of the linear theory are discussed in references [11] and [12].

\section{The Calorimeter}

The calorimeter and its constant-temperature surroundings are shown in a schematic vertical section in figure la. Figure $1 \mathrm{~b}$ is a photograph of the calorimeter as used. The apparatus is roughly cylindrical and symmetrical about a horizontal axis. The calorimeter proper, in which the laser beam is absorbed, consists of a main copper cylinder with a conical mirror on one end and a small cone closing the other. The mirror cone has a half-angle of $25^{\circ}$ and accommo-

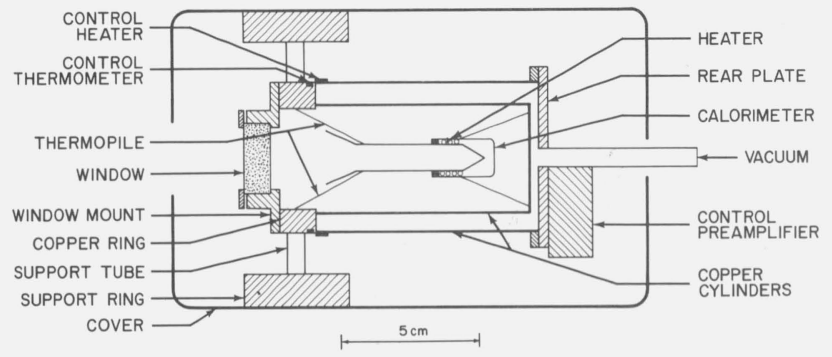

Figure la. Diagram of C3 calorimeters.

Laser radiation enters through the window and is absorbed inside the calorimeter. The temperature is measured by an 8 -junction thermopile. Electrical calibrations are carried out by applying a voltage to the heater in the calorimeter.

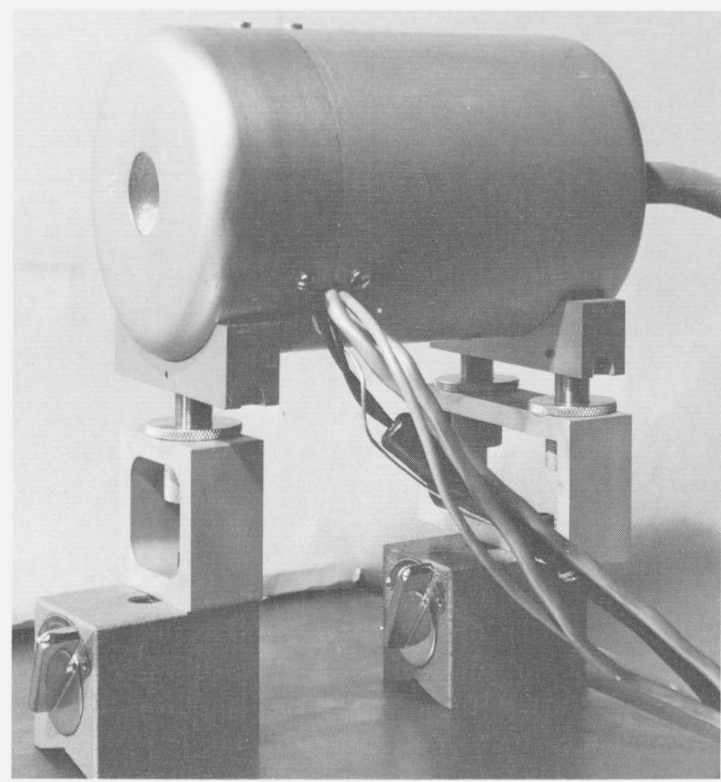

Figure 1b. Photograph of a calorimeter of the C.3 design.

dates a collimated-beam $2 \mathrm{~cm}$ in diameter by reflecting the outer part of it into the calorimeter. The small cone and the cylinder are blackened inside, by oxidizing the copper in C3-1, for example, to provide a good absorber that is reasonably resistant to high energy or power in the laser beam. This cavity construction avoids most of the error caused by variation of the absorptivity over the surface of the absorber. The outer surface of the calorimeter is gold-plated for corrosion resistance and low emittance for radiant heat transfer. The closed end, where most of the radiant energy is absorbed, is fitted with a thin copper shield in the form of a cylinder soft-soldered to a copper ring around the main cylinder. A calibrating electrical heater is wound under this shield. The shield serves to make the laser and electrical sources "equivalent" for evaluating heat transfer from the calorimeter. The problem with accounting for heat transfer is that the calibrating and laser sources cannot be in exactly the same geometrical locations and, therefore, will set up different temperature gradients. Although this geometrical effect can never be eliminated, it can, in principle, be made as small as desired. This principle is discussed in reference [11]. Application to our particular case is discussed below. The length of the cylinder represents a compromise between total absorption of radiation and the time required to make a measurement. A longer cylinder will absorb a larger fraction of the incident radiation, but the time required is increased roughly as the square of the length.

The calibrating heater is about $100 \Omega$ of \#40 manganin wire (.075 $\mathrm{mm}$ diameter) wound bifilarly to cancel inductive effects. The heater current leads are \#36 copper $(0.12 \mathrm{~mm})$ about $8 \mathrm{~cm}$ long. Potential leads are attached midway along the current leads between the surface of the calorimeter and the inner surface of the surroundings. This construction avoids possible sig- 
nificant systematic errors $[14,15]$ in comparing electrical energy to laser energy. The heat generated in the leads is 0.2 percent of the heat generated in the calorimeter. We estimate that the potential leads are located so as to allocate this 0.2 percent to better than $1 / 20$ of itself $(8 \mathrm{~mm}$ of wire in $16 \mathrm{~cm}$ of leads) for a maximum systematic error of 0.01 percent of the energy measured.

The calorimeter is suspended in the constanttemperature surroundings by an 8-junction thermopile of \#36 alloy wires $(0.12 \mathrm{~mm})$. The thermopile output is approximately $600 \mu \mathrm{V} / \mathrm{K}$. Pure metals were avoided for the thermopile because of their greater thermal conductivity and the consequent increase in the cooling constant.

The space between the calorimeter and its surroundings is evacuated to about $10^{-3}$ Torr $(0.1$ pascal $)$ to reduce heat transfer and decrease the cooling constant. The smaller cooling constant makes the internal (stored) energy of the calorimeter about four times as large as the heat exchange term. On the basis of a few experiments made at pressures higher than usual we believe that the precision of the measurement is improved by reducing the pressure and that smaller power and energy can be measured. This observation is in accord with the practice in very accurate $(0.01$ percent $)$ isoperibol calorimetry of making the heat exchange term about 1 percent of the internal energy [13].

The constant-temperature environment consists of a copper ring and two coaxial copper cylinders soldered to the ring. The inner cylinder is closed at the rear, but has four holes through which four junctions of the thermopile are drawn taut. The outer cylinder is closed by a rear plate with an "O" ring seal. A window, which in this case is a $1^{\circ}$ wedge of borosilicate glass BK7, is mounted on the front of the constant-temperature surroundings. The wedge virtually eliminates possible problems with interference. It is mounted so that a line from the thinnest part to the thickest part is nearly horizontal. Vacuum seals for the window mount are made with "O" rings.

The constant-temperature surroundings are mounted in a heavy support ring by means of three thin-wall stainless steel tubes $0.6 \mathrm{~cm}$ in diameter. Aluminum covers are fastened to the support ring. Thermopile and heater leads are brought out through the support ring. This construction allows easy access to the inner parts of the calorimeter.

\section{Temperature Control}

The temperature control of the surroundings is a critical part of the measurement. The theory treats a temperature constant in time. If the temperature varies appreciably this will cause two errors: (1) The heat exchange term will be incorrectly determined, because no allowance is made for temperature variation. (2) The internal energy will be incorrectly evaluated, because of an error in temperature measurement. The internal energy is proportional to the temperature difference $T_{F}-T_{I}$. Since the thermopile with which we determined $T_{F}$ and $T_{I}$ has its reference junctions on the surroundings, a change in the reference junction temperature between the two temperature observations will appear directly as an error in the difference.

The controlled temperature is sensed by a resistance bridge as described by Maier [16], wound in the copper ring. The bridge consists of alternate arms of copper and manganin of about $100 \Omega$ resistance fastened to the ring with an epoxy resin which cures at $100^{\circ} \mathrm{C}$. The bridge balances at about $33^{\circ} \mathrm{C}$. The bridge supply is $2 \mathrm{~V}$ DC. The output is amplified by an operational amplifier having a low offset voltage and gain stabilized with a feedback resistor. The amplified signal is fed to intermediate operational amplifiers which provide proportional and integral (reset) control to an output transistor. This transistor regulates the current in a $30-\Omega$ control heater wound near the control thermometer on the outer surface of the surroundings.

The temperature is controlled to $\pm 0.1 \mathrm{mK}$ as calculated from the gain of the operational amplifier and the bridge parameters. Our experience indicates that our temperature control is not the limiting factor in the precision of the measurement.

The temperature-controlled surroundings does not form a complete thermal enclosure for the calorimeter. Some heat is transferred by radiation through the window, although it is opaque to most roomtemperature radiation, which is a maximum at $10 \mu \mathrm{m}$. To this small extent the room is a part of the thermodynamic surroundings. In principle, the variation in room temperature would set a lower limit on the laser energy to be measured accurately in isoperibol calorimeters, but we have not yet established such a limit. Other factors are probably more important at the present time.

\section{Operation of the Calorimeter}

The output of the thermopile, which measures the difference between the temperature of the calorimeter and that of the temperature-controlled surroundings, is amplified by a D.C. amplifier linear to 0.01 percent, having a maximum gain of $10^{5}$ at $100 \mu \mathrm{V}$ full scale. The output of the amplifier $(10 \mathrm{~V} \max )$ is read by a digital voltmeter. At selected equal intervals of a few seconds, the voltmeter reading is transferred to a data coupler and then printed by a typewriter which simultaneously punches a paper tape.

In preparing for an experiment, the D.C. amplifier is allowed to warm up while the temperature control is established. About 15 min after the temperature of the surrondings is under control the rate of change of the calorimeter temperature in equation (2) becomes small enough to make a measurement. It is not unreasonable to wait until it is zero or nearly so, but it need not be zero. We keep the rate of change in the initial rating period less than about ten percent of the rate of change in the final rating period, because we believe that the increase in internal energy can be measured more accurately than heat exchange. If the calorimeter is 
cooling rapidly in the initial rating period, the heat exchange term for the experiment will be large.

The experiment cannot be started until equation (2) is obeyed, but 15 min will usually prove more than adequate for this purpose. In any case, this point is checked in the computer data analysis.

When one is satisfied that equation (2) holds, a number of data points are logged (20 to 50) and then the electrical or laser input is made. We frequently $\log$ a minimum of 20 points at $4 \mathrm{~s}$ intervals (80 s) After the input enough points are taken to make sure that 20 to 50 points are in the final rating period when higher order exponentials have become negligible and equation (2) again holds. The final rating period can usually be started about 40 sec after the input is stopped. The computer program [12] prints deviations of individual points from the integral form of the equation (2) to show whether the rating period was started too soon. If it was, the calculation is merely repeated with a later set of points.

\section{Electrical Calibrations}

The electrical calibrating circuit diagram in figure 2 is based on principles long in use in calorimetry. The diagram is included to facilitate discussion of the errors in the measurement. Electrical calibrations are carried out using the calorimeter heater as a fourterminal resistor, measuring the D.C. current in the heater, the voltage across it, and the time the power is on. The heater current is determined from the voltage across a standard resistor. Two different standard resistors are used to accommodate different heater currents. The voltages are measured with digital voltmeters. These voltmeters and the standard resistor were calibrated by the RF Power, Current, and Voltage Section of NBS.

The power source for calibration is a D.C. supply operating up to 12 volts and 0.3 amp regulated to 0.1 percent. This regulation is a matter of convenience; the voltages required for the power computation are measured. The heater power is varied by changing the fixed value series resistor.

The time is read by a counter-timer with a built-in crystal-controlled oscillator. The oscillator is checked against a $100 \mathrm{kHz}$ standard frequency supplied from the Time and Frequency Division of NBS. The timer is connected in parallel with the calibrating heater and is triggered by the voltage across the heater. Any leakage current in the timer is by-passed around the standard resistor to avoid a systematic error in the heater power.

We consider the errors in the electrical calibration by returning to equation (1). Abbreviating the corrected temperature rise by $\Delta T_{c}$, we write the electrical work $W_{e l}$ done on the calorimeter

$$
W_{e l}=E \Delta T_{c}
$$

The electrical work is the product of the voltage $\mathrm{V}_{h}$ across the heater, the current given by the voltage

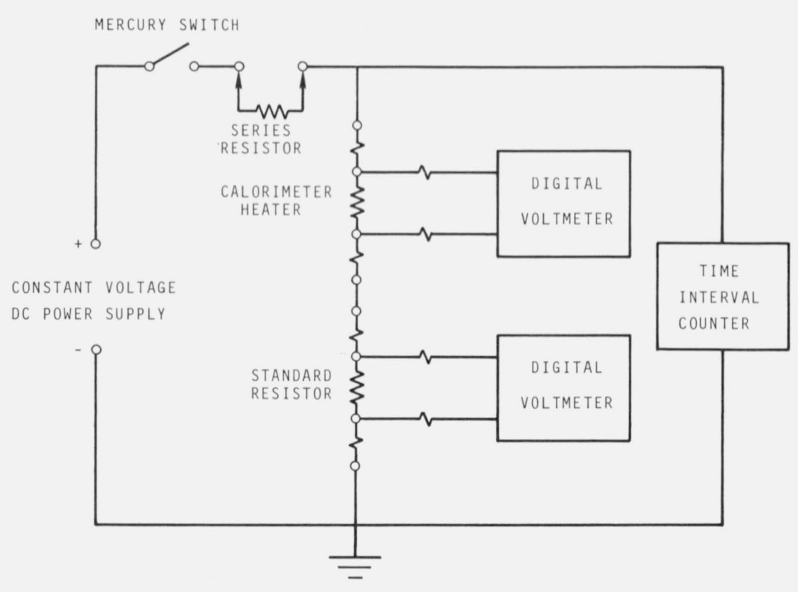

FigURE 2. Circuit for electrical calibration.

Electrical work is the product of the voltage across the heater, the current, which is the voltage across the standard resistor divided by its resistance, and the time. Any current in the timer circuit by-passes the standard resistor.

$\mathrm{V}_{r}$ across the standard resistor divided by its resistance $R$, and the time $t$. Substituting for $W$ and solving for $E$

$$
E=\frac{\mathrm{V}_{h} \mathrm{~V}_{r} t}{R \Delta T_{c}}
$$

The uncertainty in $E=f\left(\mathrm{~V}_{h}, \mathrm{~V}_{r}, t, R, \Delta T_{c}\right)$ due to systematic errors in the quantities $\mathrm{V}_{h}, \mathrm{~V}_{r}, t, \mathrm{R}$, and $\Delta T_{c}$ may be evaluated in the following manner. The systematic error $\delta E$ in $E$ caused by a systematic error $\delta \mathrm{V}_{h}$ in $\mathrm{V}_{h}$ is approximately

$$
\delta E=\frac{\partial f}{\partial \mathrm{V}_{h}} \delta \mathrm{V}_{h}
$$

However $\delta V_{h}$ is unknown except that evidence has been presented that $\delta \mathrm{V}_{h}$ is limited by $\pm d \mathrm{~V}_{h}$. It follows that the error $\delta E$ in $E$ due to $\delta \mathrm{V}_{h}$ is limited by $\pm\left|\frac{\partial f}{\partial \mathbf{V}_{h}}\right| d \mathbf{V}_{h}$. Similarly, limits on the errors in $\mathbf{E}$ due to the other four parameters may be obtained. If the error sources are uncorrelated it is clearly unlikely that they will affect $E$ in the same direction and at their extreme magnitudes. However it is conservative, and customary for a small number of error sources, to add the limits directly. Expressed as a fraction, the resulting limit to the systematic error in $\mathrm{E}$ is given by:

$$
\frac{d E}{E}=\left|\frac{d \Delta T_{c}}{\Delta T_{c}}\right|+\left|\frac{d \mathrm{~V}_{h}}{\mathrm{~V}_{h}}\right|+\left|\frac{d \mathrm{~V}_{r}}{\mathrm{~V}_{r}}\right|+\left|\frac{d R}{R}\right|+\left|\frac{d t}{t}\right|
$$

A more complete discussion is given by $\mathrm{Ku}$ [17]. The systematic error in $\Delta T_{c}$ is taken to be zero. A systematic error in $\Delta T_{c}$ would imply that either the laser or the electrical experiment affects the thermopile output in some way other than by changing the temperature of the junctions. It is possible for electrical leakage to bias the thermopile output, but checks for this source of error are easily made. A strip chart recorder connected to the nanovoltmeter will respond much more 
rapidly to electrical leakage than to the slow thermal effect, so that leakage is easy to detect as an instantaneous offset. Such an offset must be distinguished from a possible inductive effect of switching, which will appear as a spike and not an offset. We do not find either an offset or a spike due to an inductive effect. The major systematic errors are in the two voltmeters. The systematic errors in the voltage measurements are estimated to be less than 0.1 percent for one voltmeter and 0.03 percent for the other. The standard resistor calibration is stated to be accurate to 0.005 percent. No correction has been made for the temperature change of the standard resistor. This quantity is extremely small and, in any case, appears as part of the standard deviation in the electrical experiments. The counter-timer is compared to an NBS frequency accurate to about one part in $10^{9}$. The trigger error was found to be less than 0.5 microsecond, which checks the manufacturer's specifications for the counter. The counter has a least count error as used of $1 \times 10^{-5}$, but this is a random error and will be taken into account in the standard deviation. We restrict the heater current to a minimum time of $1 \mathrm{sec}$, so that the estimated systematic error in the time interval due to time base, trigger error and counting error is negligible compared to the other systematic errors.

Limits to the systematic error in $E$ are \pm 0.15 percent obtained by summing the estimated limits of \pm 0.11 percent, \pm 0.03 percent and \pm 0.005 percent estimated for $\mathrm{V}_{h}, \mathrm{~V}_{r}$ and $R$ respectively. The heater lead error discussed above has been included as part of the error in $V_{h}$. The contributions of $t$ and $\Delta T_{c}$ are taken to be negligible compared to 0.15 percent.

A chronological control chart for the electrical calibrations of calorimeter No. C3-1 is shown in figure 3 . The individual determinations of the energy equivalent are plotted in the order in which they were made. The weighted average of all points is shown as a heavy dashed line. Points below $0.3 \mathrm{~J}$ were given $1 / 16$ weight because of the poorer precision. The estimated standard deviation for an individual measurement is 0.22 percent and is plotted in the lighter dashed lines.

To ascertain whether the energy equivalent changes significantly with the total energy input, the energy equivalent of calorimeter C3-1 is plotted against energy

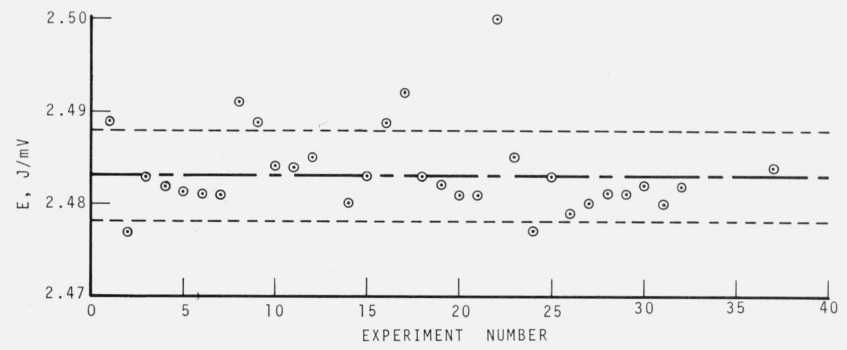

Figure 3. Control chart showing calibration factor for C3-1 versus experiment number in chronological order.

The heavy dashed line represents the weighted mean and the lighter dashed lines represent \pm one standard deviation. The calibrations cover 7 months in the development of the measurement.

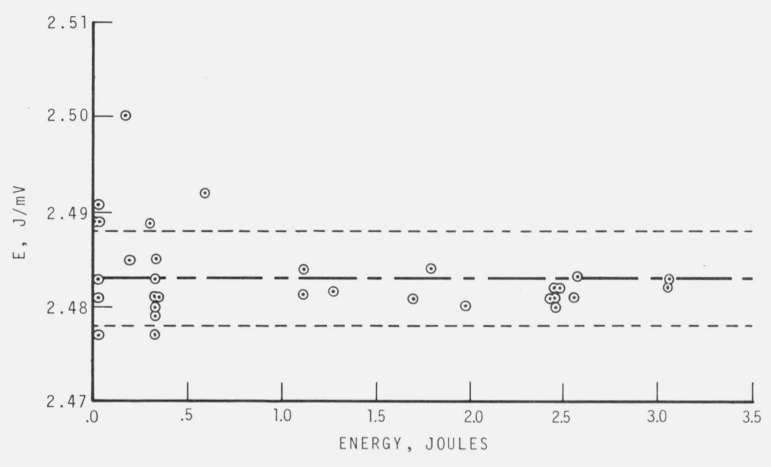

FIGURE 4. Control chart of the calibration factor for C.3-1 versus energy.

The heavy dashed line represents the mean and the lighter dashed lines represent \pm one standard deviation. Points below $0.3 J$ scatter more and were given less weight.

input in the control chart in figure 4. The lack of a significant trend with the energy input is evidence for the adequacy of the linear theory in this range. We have used the approximation that the thermopile emf is proportional to the temperature. This approximation may impair the accuracy for larger energy inputs. A few experiments at $10 \mathrm{~J}$ and $20 \mathrm{~J}$ appear to have good precision but differ a small but significant amount from the average. Present procedures sacrifice some accuracy in this range. Procedures now being developed for converting to a better temperature scale will probably extend the accurate range to these greater energies.

In a third control chart in figure 5 the energy equivalent is plotted against the cooling constant. The cooling constant is an indication of the pressure in the calorimeter; a smaller cooling constant corresponds to a lower pressure. The chart reveals no dependence of the energy equivalent on the cooling constant for calorimeter C3-1. We did find a variation of the energy equivalent of about 0.2 percent in an earlier calorimeter C2-2. While this effect can be taken into account, it is more convenient to maintain an adequately low pressure.

\section{Arrangements for Optical Intercomparisons}

A typical experimental arrangement for optical intercomparisons or calibrations is shown in figure 6 .

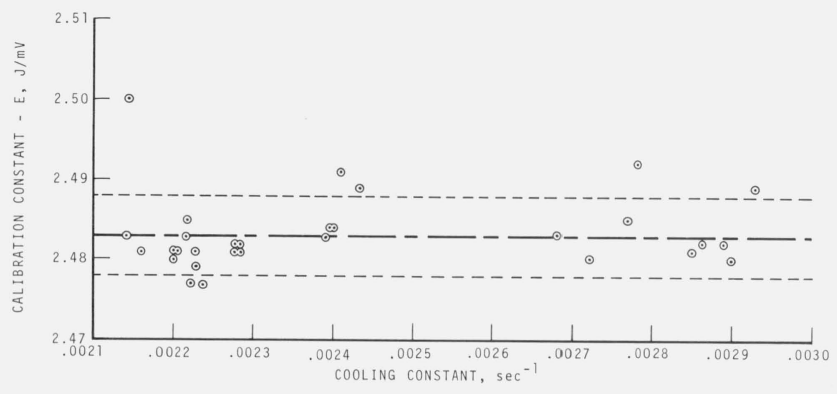

FigURE 5. Control chart of the calibration factor $\mathrm{E}$ plotted against the cooling constant to demonstrate the lack of a significant trend. 


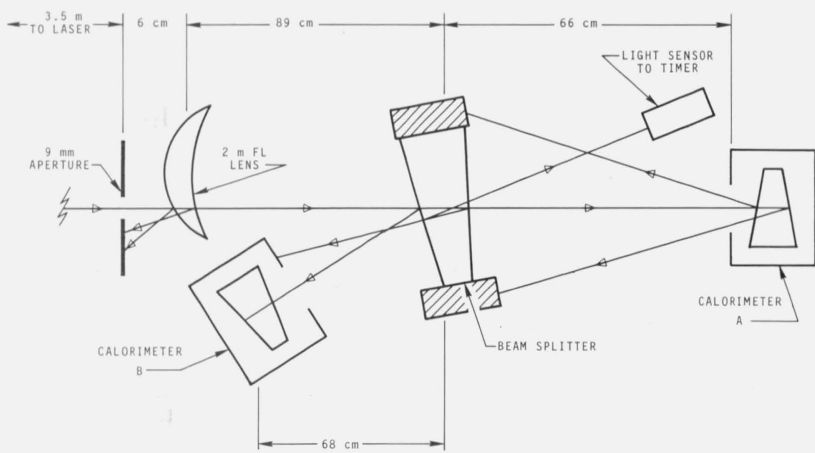

FIGURE 6. Schematic diagram showing experimental arrangement for intercomparing two calorimeters.

The aperture removes some unwanted divergent radiation. The lens reduces the beam diameter so that it will fit comfortably in the calorimeter. The first surface reflection is taken to the calorimeter at $B$. The second internal reflection from the beam splitter activates a light sensor which starts and stops a counter-timer.

In this arrangement the laser is an ion laser using argon or krypton gas. The beam is first passed through a $9 \mathrm{~mm}$ aperture stop placed about 3 meters from the exit port of the laser. The distance from the laser serves to reduce any possible effect on results of radiation due to pumping power. In this laser the pumping power is several thousand times the output power, so that a very small fraction of the pumping power could cause large errors in output power measurement. The pumping power radiation diverges rapidly, so that its effect decreases rapidly with distance from the exit port. Divergent radiation can cause a systematic error in comparison or calibration of devices having appreciably different entrance apertures or placed at different optical path lengths. Under these circumstances one device will capture more of the divergent radiation than the other. The purpose of the $9 \mathrm{~mm}$ aperture stop is to remove a small amount of extraneous laser radiation which makes an appreciable angle with the main beam. This extraneous radiation creates some confusion in setting up the experimental arrangement, but the main problem is that divergent radiation can cause systematic errors in comparison of two calorimeters having different apertures. The edge of the aperture must be kept out of any bright part of the beam to avoid diffraction effects and the corresponding divergence of the beam. The stop is painted black on both sides as a safety measure.

The beam passes next through a lens having a $2 \mathrm{~m}$ focal length. The purpose of the lens is to reduce the size of the beam so that there is no question that all of the beam enters the calorimeters. A shorter focal length lens may make the beam so small that a dust particle or imperfection in the optics may have an appreciable effect. The beam strikes the lens about $15 \mathrm{~mm}$ from the center so that reflections are carried out of the path of the main beam. The two largest reflections are absorbed on the back of the aperture stop.

The beam splitter is a $1^{\circ}$ wedge of "c" cut sapphire. Wedges are used to make it easier to sort out the various reflections and to avoid possible problems with interference. The $1^{\circ}$ angle is a compromise chosen to facilitate removal of undesired reflections without introducing problems related to the polarization of the laser beam. The laser beam strikes the first surface at an incident angle of approximately 2.3 degrees. This small angle is chosen to reduce dependence on the polarization of the laser beam. The first surface reflection is taken into calorimeter and the second surface reflection is terminated as a safety measure. The second internal reflection is taken to the silicon detector which triggers the counter-timer for timeinterval measurements.

The alignment of calorimeters of the C3 type is simple. The beam is centered on the opening in the front of the calorimeter. The calorimeter is then oriented so that the two main reflections strike the black support of the beam splitter at the level of the main beam coming through. This arrangement assures that the angle of incidence on the window is the same as that used in determinig the transmission of the window. These reflected beams are at equal angles to the main beam, so that the effects of polarization can again be neglected, and both the main beam and the second internal reflection are practically centered on the absorbing cylinder in the calorimeter.

It is important to distinguish between the first surface reflection and the first internal reflection. The distinction is not apparent to the eye, so we use the scheme shown in the diagram in figure 7 . When the two reflections straddle the main beam, the second internal reflection will be on the same side of the main beam as the first internal reflection.

\section{Window Transmission Measurements}

The arrangement for measuring the transmission of the window for C3 calorimeters is similar to that in figure 6. The calorimeter at $\mathrm{A}$ is moved to $1.1 \mathrm{~m}$ from the beam splitter and the window to be checked is placed midway between them. The window, a $1^{\circ}$ wedge, is oriented so that the two brightest reflected beams make equal angles with the incident beam, as described in the preceding section. The second internal reflection does not enter the calorimeter, as it will when

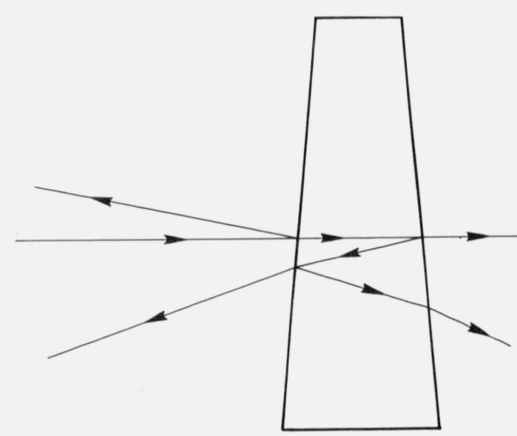

FigURE 7. Beam splitter illustrating method of distinguishing the front surface reflection and the first internal reflection.

The splitter is oriented so that the two reflections make equal angles with the incident beam. The first and second internal reflections are then on the same side of the incident beam. 
it is mounted on the calorimeter, so that the measured transmission must be increased by a few tenths of a percent, depending on the material of the window.

Since the transmission of the window is measured before it is placed on the calorimeter, a procedure has been developed which gives the measured transmission independent of the transmission of whatever temporary windows are on the calorimeters at the time.

The procedure starts with a determination of the ratio $R$ of the energy $W_{t}$ in the beam transmitted by the beam splitter to the energy $W_{r}$ in the beam reflected from the beam splitter. Using subscripts $A$ and $B$ to refer to the calorimeters in those positions, we write from equation 3

$$
R=\frac{W_{t}}{W_{r}}=\frac{E_{A} \Delta T_{A}}{\tau_{A} \alpha_{A}} \frac{\tau_{B} \alpha_{B}}{E_{B} \Delta T_{B}} .
$$

Insertion of a window in the transmitted beam will reduce the energy transmitted to the calorimeter at $A$. Using primes to allow for a possible change in the laser output, we observe the following ratio

$$
R^{\prime}=\frac{\tau_{w} \cdot W^{\prime} t}{W_{r}^{\prime}}=\frac{E_{A} \Delta T^{\prime}{ }_{A}}{\tau_{A} \alpha_{A}} \frac{\tau_{B} \alpha_{B}}{E_{B} \Delta T^{\prime}{ }_{B}}
$$

As we would predict, the ratio $W^{\prime}{ }_{t} / W^{\prime}{ }_{r}$ is observed to be independent of the laser power level, so that $W^{\prime}{ }_{t} / W^{\prime}{ }_{r}=W_{t} / W_{r}$. Substitution for this ratio in eq (5) from eq (4) gives the equation

$$
\frac{\tau_{w} E_{A} \Delta T_{A} \tau_{B} \alpha_{B}}{\tau_{A} \alpha_{A} E_{B} \Delta T_{B}}=\frac{E_{A} \Delta T^{\prime}{ }_{A} \tau_{B} \alpha_{B}}{\tau_{A} \alpha_{A} E_{B} \Delta T_{B}^{\prime}{ }_{B}}
$$

The transmission and absorption quantities for the two calorimeters appear on both sides of the equation and can be canceled. The energy equivalents $E_{A}$ and $E_{B}$ are constants, independent of the energy or $\Delta T_{c}$ over the operating range. Cancelling these and rearranging, we obtain the equation for the transmission of the window.

$$
\tau_{w}=R^{\prime} \mid R=\frac{\Delta T_{A}^{\prime} \Delta T_{B}}{\Delta T_{A} \Delta T^{\prime}{ }_{B}} .
$$

After the transmission is measured, the window is placed on the calorimeter. The transmission actually used is the measured transmission increased by a few tenths of a percent because the second internal reflection, which is discarded in the transmission measurement, enters the calorimeter when the window is mounted on the calorimeter.

Transmission measurements for calorimeter C3-1 at $676 \mathrm{~nm}$ are shown in Table 2. From these data we calculate the transmission of the window to be $\tau=0.9115$, including a correction of .0016 for the second internal reflection. The standard deviation of $\tau$ based on these measurements is 0.032 percent.

A systematic error in the transmission might arise (1) from gradual changes in the window between transmission measurements or (2) from nonuniform scatter-
TABLE 2. Beam ratio data for C3-1 window

\begin{tabular}{r|r}
\hline \hline$R$ (eq. 4) & $R^{\prime}($ eq. 5$)$ \\
\hline 11.5482 & 10.5193 \\
11.5511 & 10.5026 \\
11.5549 & 10.5044 \\
11.5436 & 10.5141 \\
& 10.5073 \\
Ave 11.5494 & 10.5077 \\
$s=0.0048$ & 10.5092 \\
\hline
\end{tabular}

ing or transmission by the glass. The first of these might come from slow formation of a coating of some kind. This kind of error can be avoided by repeating the transmission measurements at reasonable intervals. Measurements of the transmission of the window of calorimeter C3-1 made three months apart agree to 0.07 percent, although the first set of measurements was made with the calorimeter operating in air. In this case, the transmission of 0.9122 is the ratio of the energy measured with the window in its actual operating position to the energy measured with the window removed-no correction is necessary for the second internal reflection. The individual measurements are less precise without the window; the standard deviation is 0.11 percent. The 0.07 percent difference in the two values of the transmission is not significant at the 95 percent confidence level. Heasurements on another window of the same type gave the value of 0.9122 for the transmission; this value is the average of nine measurements and its standard deviation is 0.025 percent.

On the basis of the preceding information, we estimate that the systematic error in $W_{l}$ due to error in measuring $\tau$ will not exceed \pm 0.12 percent of $\tau$, or 3 times the standard deviation plus an error of .0002 in the intensity of the second internal reflection.

\section{Absorption by the Calorimeter; Excess Thermal Radiation}

It is evident from equation (3) that the fraction $\alpha$ of the incident radiation absorbed by the calorimeter must be known accurately and that the error in the fraction absorbed enters directly into the over-all accuracy of the measurement. It is also possible that there is an excess of thermal radiation from C3-1 in the laser experiment. The absorbing surface will have to run slightly hotter relative to the thermopile than in the electrical experiment because the heat flows from this surface to the metal parts of the calorimeter. Both the scattered radiation and this excess thermal radiation are taken into account in our determination of the quantity $\alpha$, which for brevity's sake we term the fraction absorbed.

The fraction absorbed by the calorimeter is measured with the experimental arrangement shown in figure 8. A small calorimeter Rl, constructed on its own window mount is placed in the evacuated calorimeter space in such a way that it intercepts practically all of the radiation, including thermal radiation, escaping from the interior of the calorimeter C3-1. 


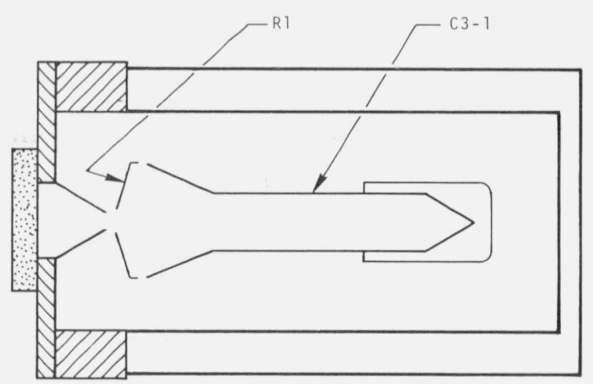

FIGURE 8. Experimental arrangement for measuring the fraction of the incident radiation absorbed.

A small laser beam passes through the aperture and through a slightly larger opening in the calorimeter $\mathrm{Rl}$ into the calorimeter $\mathrm{C} 3-1$, where most of the radiation is absorbed and measured. The small amount of radiation not absorbed by calorimeter $\mathrm{C} 3-1$ is ab sorbed and measured by calorimeter R 1 and the fraction $\alpha$ absorbed by $\mathrm{C} 3-1$ is calculated from the two measurements.

Calorimeter Rl consists of an aluminum dish $2 \mathrm{~cm}$ in diameter and about $30 \mu \mathrm{m}$ thick weighing $0.2 \mathrm{~g}$ with a $2.0 \mathrm{~mm}$ hole in the center. The dish is painted black on the surface facing calorimeter C3-1 and is mounted on three nickel-chromium alloy wires. The temperature is measured by a four-junction thermopile relative to the metal window mount, which is fastened to the temperature-controlled copper ring (fig. la). Calorimeter Rl is calibrated in a separate evacuated enclosure using a monitored $676 \mathrm{~nm}$ laser beam as in figure 6 . The mean energy equivalent from 3 experiments is $4.35 \times 10^{-4} \mathrm{~J} / \mu \mathrm{V}$ and the standard deviation of the mean is 0.6 percent. The cooling constant for $\mathrm{Rl}$ as a separate entity is $0.02 \mathrm{~s}^{-1}$.

Two types of experiments are carried out. In a laser experiment, a small beam enters calorimeter C3-1 through a $1.5 \mathrm{~mm}$ aperture and the $2 \mathrm{~mm}$ opening in calorimeter R1. The beam is focused at the aperture. In an electrical calibration, calorimeter C3-1 is heated in the usual way in order to observe the temperature change in $\mathrm{Rl}$ in response to thermal radiation from C.3-1.

The major additional problem with two calorimeters in the same enclosure as in figure 8 is that they radiate energy to one another. In the present case, thermal radiation to $\mathrm{R} 1$ from $\mathrm{C} 3-1$ is far greater than the scattered laser radiation striking $\mathrm{Rl}$ directly and the problem is to extract the desired information from the data taken in the two experiments described above.

Since the scattered radiation is a small fraction $1-\alpha$ of the incident laser radiation, this fraction can be in error by a large percent of itself without impairing the determination of $\alpha$ and hence the over-all accuracy of the calorimetric measurement of laser energy. We take advantage of this by using a simplified heat flow problem as the theoretical basis for the experiment.

In the case of an electrical calibration at constant power, the temperature of calorimeter $\mathrm{Rl}$ will rise due to thermal radiation from calorimeter C3-1. For a laser input, laser radiation will be scattered back from C3-1 and absorbed by R1 along with the excess thermal radiation. The temperature of $\mathrm{Rl}$ will rise faster than it would due to the thermal radiation alone. Typical data for the two experiments normalized to the power level of C3-1, are plotted in figure 9 . Both electrical and laser inputs were of 30 seconds duration starting at zero time.

To find the fraction $1-\alpha$ of the incident radiation received by $\mathrm{Rl}$ we treat the two calorimeters in a constant-temperature enclosure as a linear system, as in reference [11], and make use of the ideas of superposition and convolution. A constant electric power input to $\mathrm{C} 3-1$ will produce a typical timetemperature response in $\mathrm{Rl}$ because the calorimeters are thermally coupled by radiative heat transfer. A constant power $\mathrm{R} 1$ would produce a different response; the temperature would rise more rapidly for such a direct input. The time-temperature response of $\mathrm{R} 1$ to a laser input to C3-1 will be a superposition of these two responses: most of the laser beam is absorbed by C3-1, and $R l$ responds to the accompanying thermal radiation, but a small fraction of the beam will be scattered and absorbed on $\mathrm{R} 1$, giving a quicker response. The excess thermal radiation will produce a similar effect. The absorbing surface is a thin layer of copper oxide which will quickly reach a temperature excess proportional to the constant laser power, so that this component of the radiation to $\mathrm{R} 1$ would lag very little behind the input to $\mathrm{C} 3-1$. The observations therefore can account for the combined scattering of the laser beam and the excess thermal radiation. Knowing the time-temperature response of $\mathrm{Rl}$ to the thermal radiation from $\mathrm{C} 3-1$ in the electrical experiment, we

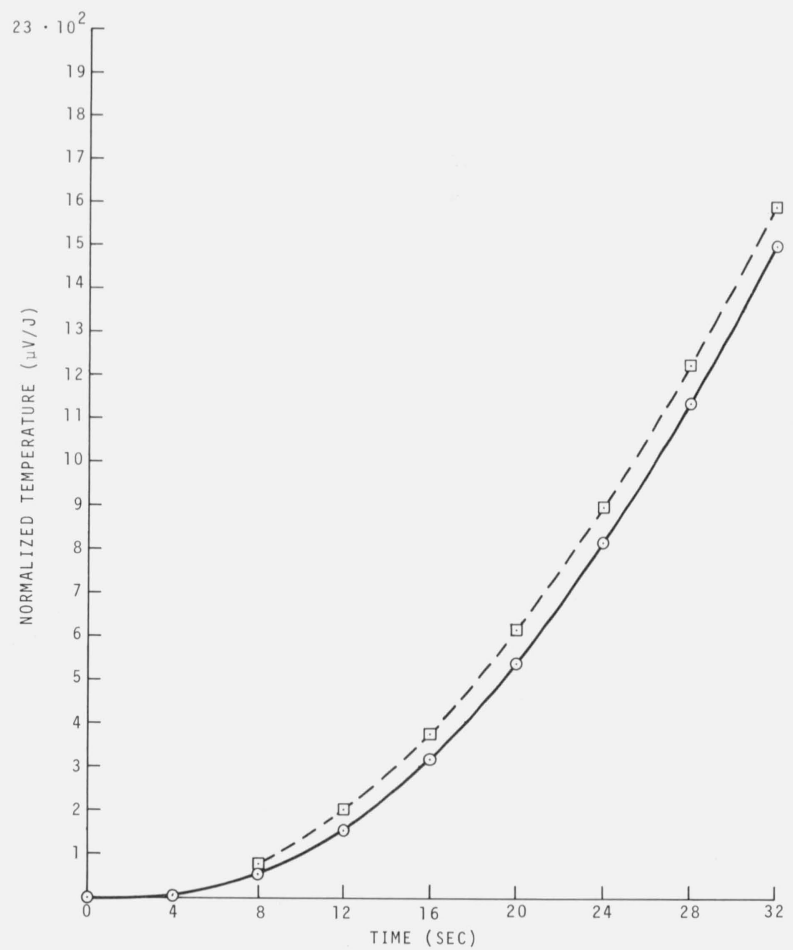

FiguRE 9. Data for finding the absorption factor for calorimeter C.3-1; comparison of time-temperature curves for calorimeter Rl.

In an electrical experiment "o" the temperature of Rl rises due to thermal radiation fn an electrical experiment "o" the temperature of $\mathrm{R} 1$ rises due to thermal radiation
from $\mathrm{C} 3-1$; in a laser experiment " $\square$ " a small fraction of the incident radiation is scattered to calorimeter $\mathrm{R} 1$ so that its temperature rises faster than in the electrical experiment. 
can subtract it from the time-temperature curve for the laser input and the remainder will be the response to the combined laser and excess thermal radiation not present in the calibration experiment.

The analysis of the resulting data is based on a simplified heat flow problem discussed by West and Churney [18] and by Churney, Armstrong and West [19]. Familiarity with their arguments will make the following discussion easier to follow. We begin by setting up an equation for the conservation of energy in calorimeter $\mathrm{R} 1$. The rate of increase in internal energy of Rl is the product of its heat capacity $\mathrm{C}$ and the rate of change of temperature $d T / d t$. When there is no direct laser input to $\mathrm{Rl}$ this increase in internal energy is equal to the rate of heat transferred to $\mathrm{R} l$. This heat transfer is the product of a heat transfer coefficient $h_{s}$ and the temperature difference $T_{s}-T$ between the constant-temperature surroundings and $\mathrm{R} 1$ plus the product of another heat transfer coefficient $h_{c}$ and the temperature difference $T_{c}-T$ between C. $3-1$ and Rl. In equation form

$$
C d T / d t=h_{s}\left(T_{s}-T\right)+h_{c}\left(T_{c}-T\right) .
$$

When $d T / d t=0$, the system is in a steady state, which is characterized by the subscript $\infty$. For this steady state

$$
0=h_{s}\left(T_{s}-T_{\infty}\right)+h_{c}\left(T_{c \infty}-T_{\infty}\right) .
$$

For calorimetry we are not interested in this steady state, so long as it is steady. Subtraction of the steadystate equation gives

$$
C d T / d t=-\left(h_{s}+h_{c}\right)\left(T-T_{\infty}\right)+h_{c}\left(T_{c}-T_{c \infty}\right) .
$$

The "temperatures" in equation (10) represent only that part of the temperature due to the laser input to $\mathrm{Rl}$; that part of the temperature due to the thermal part of the radiation has been subtracted from the composite observed temperatures before equation (10) is applied. The temperature C3-1, which has six times the heat capacity of $R 1$, will not rise much due to radiation from $\mathrm{R} 1$. The last term in equation (10) will therefore not contribute much to the temperature rise of $\mathrm{Rl}$ because $T_{c}-T_{c \infty}$ is small, so we neglect this term. With this approximation and putting $b=\left(h_{s}+h_{c}\right) / C$, we integrate equation (10)

$$
T-T_{\infty}=\mathrm{Ae}^{-b t}
$$

where $A=T_{0}-T_{\infty}$ at time $t=0$.

We now introduce the scattered laser power into the problem. Let $A$ represent the temperature due to an input to $\mathrm{Rl}$ of $1 \mathrm{~J}$ at time $t=0$; then $A=1 / \mathrm{C}$. For an input of $p d \tau$ joules at time $\tau$, equation (11) becomes

$$
T-T_{\infty}=(p / C) e^{-b(t-\tau)} d \tau .
$$

We can find the temperature due to a constant power input $\mathrm{p}$ starting at time $t=0$ by integrating equation (12) over all elapsed time $\tau=0$ to $\tau=t$ :

$$
\frac{T-T_{\infty}}{p}=\frac{1}{b C}\left(1-e^{-b t}\right) .
$$

The temperature will have a maximum or new steady state value from which we obtain the scattered laser power $p$ relative to the total power $P$ to C3-1

$$
\frac{p}{P}=b C \frac{\left(T_{\max }-T_{x}\right)}{(P \Delta t)} \Delta t .
$$

The constant $\mathrm{C}$ is obtained by calibration as described above. The cooling constant $b$ is obtained from a semi$\log$ plot of $\left(T_{\max }-T_{x}\right)-\left(T-T_{x}\right)$. In the actual case these temperatures were divided by the electrical or laser power to $\mathrm{C} 3-1$, so that we obtain the fraction of the incident light scattered to Rl. The results of four sets of paired electrical and laser experiments gave for the fraction scattered to $R 10.11$ percent, 0.17 percent, 0.13 percent, 0.12 percent (chronological order). The average is 0.13 percent. The radiation lost through the hole in $\mathrm{Rl}$ is neglected. The $2 \mathrm{~mm}$ hole is 4 percent of the total area. The painted surface of Rl absorbs about 97.6 percent of the incident radiation, and contributes a negligible error (2.4 percent of 0.13 percent). The standard deviation of the mean of these measurements is 0.02 percent.

It is possible that the absorption varies with the position in the calorimeter. To investigate this possibility we made 7 measurements of the beam ratio with a beam $3 \mathrm{~mm}$ in diameter and 8 measurements with a beam slightly over $4 \mathrm{~mm}$ in diameter. The latter illuminates about twice as large an area. The mean of the first set was 11.527 and of the second set 11.528 with a pooled standard deviation of 0.0092 . The difference is not significant, but, of course, the experiments are not sufficient to eliminate the possibility.

We estimate limits to the systematic error of \pm 0.2 percent in the fraction absorbed $\alpha$ to allow for (1) a possible error in time, which affects the positioning of the two curves in figure 9, (2) three times the standard deviation of the mean, and (3) possible variations in the fraction absorbed with the location of the beam in the absorbing cavity. This estimated systematic error must be increased if part of the radiation strikes the gold-plated conical surface.

The gold-plated conical surface will reflect most of the incident radiation into the absorbing cavity, but some will be scattered back out of the calorimeter. We compare the response of the calorimeter to radiation incident on the conical surface and directly into the cavity, but this measurement is not simply a comparison of the fractions absorbed. A basic idea of calorimetric theory is that the method of accounting for heat exchange introduces an error in the comparison of laser beams absorbed at different locations in the calorimeter-the geometric effect discussed in the next section. Also, when the calorimeter is moved so that the beam strikes in a different place, the beam comes through a different part of the window, so there may be a small effect due to imperfections in the window. Radiation striking the gold-plated cone will therefore produce a different effect because of variation in absorption, the geometric effect and the 
window. Measurements made with a $676 \mathrm{~nm} \mathrm{cw}$ laser beam $4 \mathrm{~mm}$ in diameter are shown in table 3 . The beam ratio is the ratio of the energy measured by C3 -1 to the energy measured by the monitor. The largest variation is 0.8 percent less than the ratio measured at the center of the calorimeter opening. The only change made in these experiments is movement of $\mathrm{C} 3-1$.

The conical area is not ordinarily used for intercomparisons with other devices, so this systematic error ordinarily does not affect results of intercomparisons, although some allowance must still be made for possible geometric effects. When a large beam is used, a systematic error must be estimated based on the beam size and the above measurements.

\section{The Geometric Effect}

The theory of calorimetry predicts a systematic error in the comparison of two heat sources due to the fact that the errors in accounting for heat exchange with the environment do not exactly cancel when the sources develop heat in different parts of the calorimeter. This systematic error tends to decrease as the two sources are made more remote from the surface of the calorimeter and the heat exchange is made small. Tests can be performed with known sources in different locations such as a known beam striking the calorimeter in different places or using electrical heaters in two different locations. The latter technique is more precise because it eliminates some of the uncertainties associated with small beams, such as a change in scattering by the window. Larger beams are unsatisfactory because they allow too little variation in position and they tend to average out the geometric effect. We have performed the experiments described in the preceding section to test for the geometric effect in C3-1. These tests, described in the preceding section, do not separate the geometric effect from the effects of light scattering by the gold-plated surface and by the window. The experiment was carried out with a $4 \mathrm{~mm}$ beam striking the gold-plated surface. In practice, the beam is directed at the absorbing cavity and only that part of a beam outside a $1 \mathrm{~cm}$ diameter would strike the gold-plated surface. The largest variation in Table 3 ( 0.8 percent $)$ therefore represents an unrealistically high estimate of the limits of systematic error due to the geometric effect.

TABLE 3. Beam Ratios for Light Incident on Conical Surface

\begin{tabular}{|c|c|c|}
\hline & Beam ratio & $\begin{array}{l}\text { Difference from } \\
\text { center (percent) }\end{array}$ \\
\hline \multirow[t]{2}{*}{ Centered. } & 11.532 & ......... \\
\hline & 11.525 & ......... \\
\hline \multirow[t]{2}{*}{$5 \mathrm{~mm}$ Right.. } & 11.436 & -0.8 \\
\hline & 11.433 & \\
\hline $4 \mathrm{~mm}$ Right.. & 11.468 & -0.5 \\
\hline $4 \mathrm{~mm}$ Left.... & 11.514 & -0.1 \\
\hline \multirow[t]{2}{*}{$5 \mathrm{~mm}$ Left.... } & 11.490 & -0.3 \\
\hline & 11.495 & \\
\hline
\end{tabular}

We have checked the geometric effect in an earlier version of the calorimeter C2-2 using two electrical heaters. The heaters are located at each end of the absorbing cylinder so that they greatly exaggerate the geometrical effect since a laser beam would mainly be absorbed at the closed end. The averages of six experiments with each heater are 3.614 and 3.606. The standard deviation of the mean is 0.0061 , so that the two agree within twice the standard deviation. The difference between the average values is 0.22 percent. On the basis of the geometric effect in Calorimeter C2-2 and the data in Table 3, which may possibly be due to the geometric effect, we estimate limits to the systematic error of \pm 0.5 percent of the energy measured. Note that we would have to make some allowance for a systematic error in any case because the tests that can be made are only indicative of the magnitude of the error; they do not prove that there is no possible way that a greater systematic error could not be incurred.

\section{Precision and Accuracy of a Laser Energy Measurement}

In analyzing the over-all precision and accuracy of the measurement we first examine some errors not yet considered.

Polarization. The angle of incidence of the laser beam on the calorimeter window is $0.75^{\circ}$. We calculate a maximum error of $4 \times 10^{-5}$ in the transmission due to a change from vertically polarized to horizontally polarized light.

Thermal radiation from the window. Some of the incident radiation will be absorbed by the window, raising its temperature and increasing the thermal radiation to the calorimeter over that present during electrical calibrations. This radiation will not reach the calorimeter $\mathrm{C} 3 \mathrm{-l}$ in the absorption measurements because of calorimeter R1, figure 8 , so it must be treated separately. We calculate that a $20 \mathrm{~J}$ laser input will raise the glass temperature $4 \mathrm{mK}$, assuming no heat loss from the glass. The resulting thermal radiation to the calorimeter will be less than 0.01 percent of the input.

Errors from data logging equipment. The gain stability and linearity of the data acquisition equipment does not contribute a systematic error. Short term instability will appear in the random error. If there is a long term drift it will show up on the chronological control chart for the energy equivalent, figure 3. Nonlinearity will limit the useful range of the calorimeter and will show up in the control chart of energy equivalent plotted against total energy input.

It is prudent here, as with the digital voltmeters, to utilize most of the scale of the instruments. In this way least count and percent-of-scale errors are minimized. Measurements and calibrations for a given energy should be made on the same scale.

Precision and accuracy of a measurement of a laser energy input. The error in measuring a laser input is estimated by reference to equation (3). Following the recommendations of Eisenhart [20], we present 
the systematic and random errors separately. The limits of systematic error estimated above for the various factors are: $d E / E= \pm 0.21$ percent; $d \alpha / \alpha= \pm 0.2$ percent; $d \tau / \tau= \pm 0.12$ percent; thermal radiation from the window \pm 0.01 percent; and the geometric variation $= \pm 0.5$ percent so the limits on the total systematic error are estimated to be the sum of these or \pm 1.0 percent of the measured energy. As more data become available, better, and probably smaller, limits can be placed on the systematic errors.

The random error associated with the measurement of a laser energy $W_{l}$ input to the calorimeter is a useful quantity because it is one of the criteria for judging its merits relative to other devices and its applicability to a particular problem. This random error is due entirely to the random error in determining $\Delta T_{c}$ since the other factors on the right-hand side of equation (3) are constants:

$$
W_{l}=\frac{E \Delta T_{c}}{\tau \alpha}
$$

To estimate the random error from repeated measurements on a particular laser we would have to depend on the stability of the laser, a dependence we are not willing to assume. In general, the random error is best estimated from electrical calibration data because the contribution from the electrical measurements can be negligible. In our particular case, experimental evidence indicates that the old digital voltmeters are not sufficiently stable. The standard deviation of an electrical calibration is 0.22 percent of the measured energy based on 18 determinations. The standard deviation as a percent of the measured energy increases as the measured energy decreases below about $0.3 \mathrm{~J}$ for both electrical and laser inputs, so we will confine our considerations to energies greater than $0.3 \mathrm{~J}$. The pooled standard deviation based on three sets of beam ratio experiments (19 experiments, 16 degrees of freedom) is 0.08 percent. The standard deviation of a measurement with either calorimeter by itself must, of course, be less than 0.08 percent, but in any case this 0.08 percent is so much smaller than the 0.22 percent for electrical calibrations that we conclude that the electrical measurements themselves are making a major contribution to the random error.

For lack of a better basis, we estimate the random error associated with a laser energy input from the beam ratio measurements, making a few simplifying assumptions. Since the absorption of the cavity and the window transmission were measured only for C3-1, we first assume that these quantities are the same for both calorimeters. These quantities then cancel in equation (4) and we have for the beam ratio

$$
R=E_{A} \Delta T_{A} / E_{B} \Delta T_{B}
$$

From propagation of error formulas we know that the standard deviation $s_{R}$ of determinations of $R$ can be expressed in terms of the standard deviations $s_{A}$ and $s_{B}$ of the corrected temperature rises $\Delta T_{A}$ and $\Delta T_{B}$ :

$$
\left(\frac{s_{R}}{R}\right)^{2}=\left(\frac{s_{A}}{\Delta T_{A}}\right)^{2}+\left(\frac{s_{A}}{\Delta T_{B}}\right)^{2}
$$

If we assume that the standard deviations of $\Delta T_{A}$ and $\Delta T_{B}$ are dependent on the input energy and expressible as a constant fraction, that is, if

$$
s_{A} / \Delta T_{A}=s_{B} / \Delta T_{B},
$$

then $s_{A} / \Delta T_{A}=0.0008 / \sqrt{2}$ or 0.057 percent. Assuming that $s_{A}=s_{B}$ and that they are independent of the energy input, and using $\Delta T_{A}=17 \Delta T_{B}$ from the data, we estimate the standard deviation for the measurement of a laser input to be 0.02 percent at $0.1 \mathrm{~J}$ and 0.002 percent at $1 \mathrm{~J}$. Until better electrical calibration data are available, we will use the more conservative estimate of 0.06 percent for the random error associated with a laser energy input to calorimeter C3-1.

One anticipated use for the $\mathrm{C}$ series calorimeters is in testing or calibration of other devices. For this purpose we currently use the experimental arrangement in figure 6. One calorimeter is used as a monitor (at either position) and the device to be tested is substituted at the other position. The beam ratio $R$ is given by equation (4).

$$
R=\frac{E_{A} \Delta T_{A}}{\tau_{A} \alpha_{A}} \frac{\tau_{B} \alpha_{B}}{E_{B} \Delta T_{B}} .
$$

The standard deviation of this ratio is 0.08 percent. If a device to be calibrated is now substituted for $B$ the ratio can be written in terms of the calibration factor $F$ and the scale reading $S$ of the device to be calibrated

$$
R^{\prime}=\frac{E_{A} \Delta T_{A}^{\prime}}{\tau_{A} \alpha_{A}} \frac{1}{F S}
$$

If we are careful to maintain the experimental optical arrangement applying to equation (4), then $R=R^{\prime}$ and we obtain for the calibration experiment

$$
F S=\frac{\Delta T_{A}^{\prime}}{\Delta T_{A}} \frac{E_{B} \Delta T_{B}}{\tau_{B} \alpha_{B}} .
$$

The systematic error in the monitoring calorimeter at A cancels in this equation but the random error from our measuring system is increased because there are now two contributions from the monitor in $\Delta T_{A}$ and $\Delta T_{A}^{\prime}$. The random error in the scale reading $S$ will make an additional contribution to the error in the calibration factor $F$. The point is that the precision and accuracy of any measurement made with the C3 calorimeters will depend on what additional parameters are introduced. Each measurement must be examined from this point of view and planned so as to eliminate additional errors if possible.

\section{Intercomparison of Calorimeters}

The $\mathrm{C}$ series calorimeters are being proposed as devices for measuring laser energy relative to electrical standards, so it is important to compare C3-1 to some 
of the liquid cell calorimeters which have been the main basis of NBS laser power and energy measurements $[1,3]$. For this comparison we selected two liquid cell calorimeters having laboratory designations AF2 and AF6. The ratios of the energy measured in a designated calorimeter to the energy measured by the monitor calorimeter are given in Table 4 . These ratios differ from others given in this paper because an extra lens was inserted so as to give a beam $1.1 \mathrm{~cm}$ in diameter on the liquid cells. To avoid a possible bias from interference effects, the liquid cell calorimeters were repositioned for each experiment. The means of measurements of AF2 and AF6 differ from the mean of the measurements for $\mathrm{C} 3-1$ by -2.1 percent and -1.8 percent respectively. Considering the claim of 2 percent for the "estimated calibration uncertainty" of a typical liquid cell calorimeter and the random and systematic errors considered earlier in this paper, the differences of about 2 percent seem somewhat too large. We therefore investigated two possible sources of systematic error in the liquid cell calorimeters: (1) the possibility that the small amount of energy reflected from the glass-solution interface might result in an appreciable systematic error and (2) the possibility that the geometric effect had been underestimated.

In the first series of experiments we used a $676 \mathrm{~nm}$ beam $4 \mathrm{~mm}$ in diameter and let it strike the liquid cell in the center and at distances of 4 and $5 \mathrm{~mm}$ from the center in all directions. The results are given in Table 5 in chronological order. The calorimeter was re-aligned after each measurement. The measurements have a standard deviation of 1 percent, appreciably larger than normal, but the mean differs from the value in Table 2 obtained with C3-1 by the same -2.1 percent. We conclude from these experiments that there may be a measurable interference effect if the liquid cell calorimeter is used with small beams, but that there is no appreci-

TABLE 4. Intercomparison of calorimeters

\begin{tabular}{c|r|r|r}
\hline Date & $\begin{array}{r}\text { Ratio AF2 } \\
\text { to monitor }\end{array}$ & $\begin{array}{r}\text { Ratio AF6 } \\
\text { to monitor }\end{array}$ & $\begin{array}{r}\text { Ratio C3-1 } \\
\text { to monitor }\end{array}$ \\
\hline $4-28$ & 10.538 & & 10.773 \\
10.541 & & \\
$4-29$ & 10.545 & & 10.777 \\
& 10.540 & & 10.767 \\
$4-30$ & & 10.580 & 10.765 \\
& & 10.577 & \\
$5-5$ & 10.534 & & 10.766 \\
& 10.546 & & 10.782 \\
& Mean 10.541 & 10.581 & 10.772 \\
& $\mathrm{~s}=.004$ & $\mathrm{~s}=.005$ & $\mathrm{~s}=.007$ \\
\hline
\end{tabular}

TABLE 5 Beam ratio measurements with liquid cell calorimeter

\begin{tabular}{r|l}
\hline \hline \multicolumn{1}{c|}{ Ratio } & \multicolumn{1}{|c}{ Beam location } \\
\hline 11.430 & Center \\
11.213 & $5 \mathrm{~mm}$ right of center \\
11.382 & Center \\
11.124 & $4 \mathrm{~mm}$ left \\
11.223 & $5 \mathrm{~mm}$ left \\
11.494 & $4 \mathrm{~mm}$ above \\
11.246 & $4 \mathrm{~mm}$ below \\
11.278 & Center \\
11.310 & Center \\
11.130 & Center \\
11.327 & $4 \mathrm{~mm}$ right \\
Ave 11.287 & \\
$\mathrm{~s}=0.117$ & \\
\hline
\end{tabular}

able geometric effect associated with the distribution of energy anywhere on the exposed surface of the window.

There is another possible systematic error due to the geometric effect in an axial direction, which comes about because ${ }^{-}$heat generated by the electrical heater is closer to the silver housing than heat generated by a laser beam. In an electrical calibration the thermocouple located on the silver housing responds very quickly [1] but the glass surface will run colder during the heat input because heat must reach the glass through poor thermal conductors. In a laser experiment, the beam is mostly absorbed in the first millimeter of liquid behind the glass and the heat generated must flow to both the glass and the housing through poor thermal conductors. The net effect is that the integral $\int T d t$, which accounts for the heat exchange, is overestimated in an electrical experiment relative to a laser experiment and the resulting energy equivalent calculated from equation (1) is too small.

To get some estimate of the magnitude of this effect, we have taken a liquid cell calorimeter of an earlier design with an extra thermocouple mounted on the center of the front glass surface. An electrical calibration was carried out and the time-temperature data were taken with both thermocouples. The corrected rise was calculated for each of them by equation (1). The results differed by 3.6 percent. Since the thermocouple on the housing overestimates the heat exchange and the thermocouple on the glass underestimates it, the true value lies in between. Qualitatively, this is in the direction required to reconcile the differences between the liquid cell calorimeters and C3-1.

We are grateful to Peter $\mathrm{V}$. Tryon for help with analysis of the data and discussions of the errors and to Barbara E. Orr for most of the computations. 


\section{References}

[1] Jennings, D. A., West, E. D., Evenson, K. M., Rasmussen, A. L., and Simmons, W. R., NBS Technical Note 382 (1969).

[2] Jennings, D. A., and West, E. D., Rev. Sci. Instr. 41, 565 (1970).

[3] Jennings, D. A., IEEE Transactions on Instr. and Meas. IM 15, 161 (1966).

[4] Birky, M. M., Applied Optics 10, 132 (1971).

[5] Pontius, P. E., and Cameron, J. M., NBS Monograph 103 , U.S. Government Printing Office, Washington, D.C. (1966).

[6] Pontius, P. E., NBS Technical Note 288, U.S. Government Printing Office, W ashington, D.C. (1966).

[7] Experimental Thermochemistry, F. D. Rossini, ed., (Interscience Publishers, Inc., New York, 1956).

[8] Experimental Thermochemistry, Vol. II, H. A. Skinner, ed., (Intersicence Publishers, Inc., New York, 1962).

[9] Experimental Thermodynamics, Vol. I, (J. P. McCullough and D. W. Scott, ed., Plenum Press, New York, 1967)

[10] Roth, W. A., and Becker, F., Kalorimetrische Methoden zur Bestimmung Chemischer Reaktionswarmen, F. Vieweg and Son, Braunschweig, Germany (1956).
[11] West, E. D., and Churney, K. L., J. Appl. Phys, 41, 2705 (1970).

[12] West, E. D., NBS Technical Note 396, U.S. Government Printing Office, Washington, D.C. (1971).

[13] Coops, J., Jessup, R. S., and Van Nes, K., in Experimental Thermochemistry, F. D. Rossini, ed., (Interscience Publishers, Inc., New York, 1956).

[14] Ginnings, D. C., and West, E. D., Rev. Sci. Instr. 35, 965 (1964).

[15] West, E. D., and Ishihara, S., Rev. Sci. Instr. 40, 1356 (1969).

[16] Maier, C. G., J. Phys. Chem. 34, 2860 (1930).

[17] Ku, H. H., Precision Measurement and Calibration, NBS Special Publication 300, Vol. I, U.S. Government Printing Office, W ashington, D.C. (1969) p. 331.

[18] West, E. D., and Churney, K. L.. J. Appl. Phys. 39, 4206 (1968).

[19] Churney, K. L., Armstrong, G. T., and West, E. D., Status of Thermal Analysis, NBS Special Publication 338, U.S. Government Printing Office, Washington, D.C. (1970).

[20] Eisenhart, C., in ref. 17, p. 21.

(Paper 76Al-694) 